\title{
"Into the Unknown" Supervising Teacher Candidates During the 2020 COVID-19 Pandemic
}

\author{
Steffany Maher \\ Indiana University Southeast \\ stmaher@iu.edu \\ Alan Zollman \\ Indiana University Southeast \\ alanzoll@ius.edu
}

\begin{abstract}
In mid-March 2020, our public schools ended classroom instruction because of the 2019 coronavirus disease (COVID-19) pandemic. The timing of the suspension of face-to-face instruction was in the middle of the student teaching clinical experience for our secondary education teacher candidates. Without preparation, teacher candidates were to guide their middle and high school students through online learning. University faculty were experiencing a similar challenge— how to support and direct their teacher candidates in midexperience. This was a change in the logistics of teaching and in the focus of education. Unlike in previous years, the first priority of schools was not high-stakes standardized testing, nor daily pacing guides, but rather the emotional and social health of students. This change in the schools' priorities fit well with the preparation of our teacher candidates at our Midwest regional teaching university. Our focus was to prepare teachers of students, not teachers of mathematics or English. While our secondary teacher candidates did not have all the tools and technological skills needed to switch to online teaching immediately, they did know it was the relationship with the learner that was most important. The second change in the schools' priorities was a time-allocation switch from mostly teaching to mostly planning, communicating, and supporting one another as teachers. Our teacher candidates already knew that effective communication makes everyone a better instructor and benefits student learning. To help our teacher candidates make these transitions, we developed and used a clinical practice interview protocol with our candidates regularly online. In addition to these changes in priorities in schools, another helpful experience our teacher candidates possessed was prior experience as students of online classes themselves. Many had taken several online or hybrid university courses and knew what worked well and what they needed to avoid as online teachers. In this reflective essay, we discuss how the university academic clinical educators (university supervisors) supported and facilitated our teacher candidates in preparing and implementing quality instruction during the COVID-19 pandemic.
\end{abstract}

Keywords: teacher candidates, COVID-19 pandemic, teacher supervision, student teaching, clinical practice.

\section{The 2019 Coronavirus Disease Crisis}

In this reflective essay, we discuss how we, the university academic clinical educators (university supervisors), supported and facilitated our secondary education teacher candidates (student teachers) in preparing and implementing quality instruction through the 2019 coronavirus disease (COVID-19) pandemic. What began as an extended spring break for P-12 (prekindergarten to 12th grade) and postsecondary schools turned into a complete shutdown when, in mid-March of 2020, public schools in our Midwest state ended classroom instruction because of the pandemic. This suspension of face- 
to-face instruction took place in the middle of our secondary education teacher candidates' clinical experience. Thus, without any preparation, our teacher candidates were asked to guide their middle and high school students through online learning. Our university teacher educators were experiencing a similar challenge: How do we support and direct our teacher candidates, midexperience, through this transition to distance teaching?

\section{COVID-19's Effect on National Education Priorities}

As COVID-19 spread across the United States, we began to see a change in the logistics of teaching and in the focus of education in general. The first priority of schools all over the nation was not highstakes standardized testing, as it had been before the pandemic (we saw states cancel these tests), nor daily pacing guides through scripted curriculum, but rather the emotional and social health of students. As our focus at our university was to prepare teachers of students rather than teachers of mathematics or English, this change fit well with the preparation of our teacher candidates. While our secondary candidates did not have all the tools and technological skills needed to switch to online teaching immediately, they did know it was the relationship with the learner that was most important.

\section{Our Approach to the Crisis}

Our approach was to supervise and support our teacher candidates following the three stages of newteacher development described by Griggs, Sullivan-Losey, and Zollman (2018). They proposed that new teachers and teacher candidates progress through three stages of development: first being concerned about oneself, next being concerned about the subject content, and last being concerned about their students' learning. For example, teacher candidates' first priority is themselves: Where should I park, which bathroom should I use, how should I dress? In a non-COVID-19 year, this stage is fairly short in duration. When these concerns are allayed, the candidates move to a focus on the subject content. Some candidates (and teachers) never progress beyond this stage. They view themselves as teachers of the subject, not teachers of students. When candidates are secure in the content knowledge they are teaching, they move to a focus on their students' learning of the content.

In our initial approach to teacher candidates after the school shutdown, we communicated with our candidates on this first stage of new teacher development, concerns about oneself. We checked in with our candidates regularly, asking how they were doing physically and emotionally. We also followed Abraham Maslow's Hierarchy of Needs (1954) in our remote communications strategy. Maslow's hierarchy describes a person's most fundamental needs as physiological, followed by safety, belongingness and love, esteem, and ultimately, self-actualization. Although there is criticism of the original hierarchy's argument that a lower level must be completely satisfied before one moves onto a higher level, this model is widely used in business and education motivational training. We believed it would be useful for our students.

For our purpose of connecting and communicating with our teacher candidates, we found this hierarchy to cover most of our topics in a sequential process. In fact, we developed our own clinical practice interview protocol (see Appendix), based on both Griggs et al.'s (2018) new teacher development stages and Maslow's (1954) hierarchy.

Thus our first response as teacher educators was to reach out to our students- to offer reassurance that they would get through this and that they would be able to graduate on time. This correlates with concerns about oneself as a teacher and with Maslow's (1954) need for safety. This initial reaction also stemmed directly from our philosophy of teaching. At our university, our teacher educator program believes in educating the whole child. We teach the student, not the content.

Journal of Teaching and Learning with Technology, Vol. 10, Special Issue, jotlt.indiana.edu 
Our second focus was to help alleviate our teacher candidates' concern about the subject content. Our teacher candidates transitioned from mostly teaching in their classrooms to mostly planning, communicating, and supporting one another. It was at this point, to successfully monitor this transition, that we developed a formal clinical practice interview protocol and asked our candidates to complete it at intervals during their distance-teaching experience and share it with their university academic clinical educator. For this stage, we built on the foundation our candidates had with their own online education and encouraged use of the technology available to them.

Throughout this ever-evolving pandemic experience, our third and ultimate priority was to care for our teacher candidates as best we could from a distance and to ensure their development, as we were concerned about our students' learning (Griggs et al., 2018). We modeled this to our teacher candidates in our education methods courses, encouraging them to embrace this philosophy of education in their own teaching practices.

Meeting with our secondary teacher candidates through the video conferencing tool Zoom was one way that we checked in with them to ensure they were staying physically, mentally, and emotionally healthy, had the basic content knowledge, and focused on their students. During these meetings we were pleased to hear our candidates sharing how they were checking in with their own students. They often were worried about students with whom they were not able to connect during their distance teaching. As teacher educators, we were pleased to see our candidates focused on their students, rather than themselves, the content, test scores, or curricula. They shared our ultimate priority in education.

\section{Our Teacher Candidates' Personal Challenges}

As educators ourselves, we also worried about our candidates. Some of them were living at "home" in states apart from where they were teaching; some had limited access to Wi-Fi; others struggled to stay connected with their P-12 clinical educators (mentor teachers) and their students; one served in the National Guard and was called up for active duty while completing his clinical practice through distance teaching. Our weekly Zoom "check-in" meetings were a vital part of staying connected, troubleshooting problems, and continuing to live in relationship with one another from a distance.

Many of our students worked part-time in the service industry, for instance, as waitstaff in restaurants. These jobs disappeared when the state ordered mandatory closure of nonessential businesses. Money for food and rent disappeared. To respond to the psychological and safety needs of our students, the university initiated a Student Emergency Assistance Fund, supported by faculty and staff. We publicized the availability of these funds to our teacher candidates. Several candidates applied and were awarded monies - within just a few days. Along with the Assistance Fund, the university's Grab \& Go Emergency Food Pantry provided nonperishable food items, as well as gift cards to grocery stores and gas stations, to our candidates in need.

\section{Our Teacher Candidates' Transition to Distance Teaching}

Similar to the change in the public schools' priorities was our candidates' time-allocation switch from mostly teaching to mostly planning, communicating, and supporting one another as teachers. Our teacher candidates already knew that effective communication makes everyone a better instructor and benefits student learning. To help our candidates make this transition, we developed the clinical practice interview protocol and met with them weekly online. The protocol asked our teacher candidates questions about their distance-teaching clinical practice experiences. The completed protocol and subsequent Zoom meetings took the place of our final two formal observations of their 
teaching. The protocol questions were divided into three categories: Logistics, Teaching, and Closure, following our teaching philosophy.

One aspect the protocol revealed was a feeling of disconnect from us and their fellow teacher candidates. Additionally, our candidates had been developing relationships with their P-12 clinical educators and students and wanted to continue to foster those relationships, even if they had to do it from a distance.

To help with this dilemma, educators all over the United States were quickly learning how to teach online synchronously through mediums such as Zoom and Google Meets, another videocommunication platform. Most of our candidates had never taken part in a Zoom meeting before. Thus, our university academic clinical educators intentionally modeled how to conduct an online class meeting and student check-in via Zoom, skills that were easily transferable to other platforms, such as Google Meets.

Most of our candidates engaged with Google Classroom, as this was their placement schools' online learning platform. Some candidates were able to teach online along with their classroom teachers because their school provided access. Others had to send their lessons and materials to their P-12 clinical educators because their school did not provide access to them as teacher candidates. Whatever access our candidates had to their students, we did our best to guide them through distance teaching. In fact, the university set up internet "hot spots" for our students. Similarly, several of the school districts, using school buses, did the same for their students to have internet access.

As part of the university system in our Midwest state, our university offers free download use of Kaltura for video creation, and several of our candidates utilized this in their teaching. Our candidates also were well versed in Kahoot and other online "game" applications, such as Quizizz and Quizlet, to keep students motivated and connected. Our teacher candidates also used these apps for formative and summative content assessments. In addition to these apps, for content-specific teaching, as in mathematics, our teacher candidates needed to learn and then use such programs as EquatIO, Jamboard, GeoGebra, Symbolab, Photomath, and WolframAlpha.

In a change from "normal" interactions with teacher candidates, the university faculty set up text messaging through Remind, a messaging service built especially for education. In this venue, the teacher candidates felt comfortable in communicating concerns, fears, depression, and content questions directly with us. It also made it possible for us to quickly and privately check in with our teacher candidates, asking them about their specific circumstances and caring for them from a distance.

\section{Our 'Teacher Candidates' Advantages}

While our candidates had much to learn in this abrupt transition, they also had several unique advantages. One was that our teacher candidates had been in the classroom with their P-12 clinical educators during the fall semester before clinical practice. They had an established rapport with their classroom teachers, and these teachers trusted our teacher candidates as co-teachers in planning and teaching. Several teachers allowed our candidates to take the lead in the online teaching.

A second advantage was the rapport our teacher candidates felt among themselves as a cadre. They shared fears, frustrations, ideas, and assistance. They felt a responsibility to support one another through this crisis. This was evident in our Zoom meetings and in the protocol.

A third advantage was teacher candidates' prior experience as students of online classes themselves. Many had taken several online or hybrid university courses and knew what worked well and what they needed to avoid as online teachers. As mentioned previously, our candidates understood that building relationships with students to foster student learning was the ultimate objective.

Journal of Teaching and Learning with Technology, Vol. 10, Special Issue, jotlt.indiana.edu 


\section{Final Reflection}

What did we learn from this experience? Reflecting upon the semester, we, as university academic clinical educators, successfully stayed focused on our philosophy of preparing teachers of students, not teachers of mathematics or English. While our secondary teacher candidates did not have all the tools and technological skills needed to switch to online teaching immediately, they did know it was the relationship with the learner that was most important.

Throughout the semester, the main concept that we reinforced during the crisis was the importance of trust and respect. Our teacher candidates must establish a rapport of trust, respect, and communication with their students; our candidates must establish a similar rapport with their P-12 clinical educators; and we must establish a rapport with our candidates. With distance learning, this is challenging.

Our candidates learned that it is important to take the time during Zoom and Google Meets meetings to include activities, such as ice breakers, that develop rapport. Through these activities, teachers create community with their students, even in online spaces, so students see by their actions that teachers value them as individual learners.

Our teacher candidates faced an unprecedented crisis while finishing their education degree. Because of our rapport with our candidates, while they struggled, they maintained the teaching objective of educating the whole student. They did not view themselves as online teachers-they viewed themselves as teachers of students. We finished their clinical experience pleased with how our candidates approached this crisis, and we believe this bodes well for them as future teachers in the ever-evolving field of education.

\section{Appendix}

\section{Appendix 1. Secondary Education COVID-19 Clinical Practice Interview Protocol.}

We understand that these are strange times and that you are working with your P-12 Clinical Educator to offer the best learning opportunities possible for your students. Because you are all teaching from a distance, we have developed this protocol in order to best understand your teaching practices and clinical experiences for the remainder of your clinical practice. Please answer each question to the best of your ability. Once complete, please email this to your Academic Clinical Educator. Your Academic Clinical Educator will review it before meeting with you via Zoom to discuss your experiences.

We plan to use this protocol and the subsequent Zoom meeting to take the place of our two remaining observations of your clinical practice. Please contact your Academic Clinical Educator with any questions.

Part A. Logistics

1. How many weeks of clinical practice have you completed?

2. Is your school using E-Learning, packets, or both to teach from a distance?

3. If so, since we last observed, please explain how you are expecting to use these tools for teaching and learning?

a. Planning:

i. How will you co-plan or plan with your P-12 Clinical Educator?

ii. What resources are you using to create meaningful learning experiences?

b. Instruction:

i. How will you deliver the instructional material in a meaningful way?

ii. What will be the process for delivering the material?

Journal of Teaching and Learning with Technology, Vol. 10, Special Issue, jotlt.indiana.edu 
c. Evaluation:

i. How will you know if this material has created knowledge?

ii. Formative/ Summative?

d. Experience:

i. How will you make this a meaningful experience for your students, who are already suffering from angst due to the disruption of everyday life?

4. If not, how do you and your P-12 Clinical Educator plan to teach from a distance? Please explain in as much detail as possible.

5. How much of YOUR unit were you able to teach before your school changed to distance learning? Were you able to get data to use in the Student Learning Project? What is the status of this project?

6. What ideas do you have for showing student growth if you were not able to complete the datagathering portion of a unit (Pre-assessment, post-assessment), either with a unit you created or with a section of distance learning? Feel free to brainstorm ideas and ask questions here:

7. Has anything changed since we last spoke to one another? If so, what is new?

Part B. Teaching

Please email your lesson plan along with this completed form and any questions you may have for your Academic Clinical Educator.

1. How are you and your P-12 Clinical Educator adjusting to changes?

2. How much contact do you have with your students? What efforts are you making to encourage students in their distance learning?

3. How are you assessing your students' work?

4. What data do you plan to gather to show student growth? What data have you already gathered?

5. Have you been able to upload any of the Taskstream assignments (e.g., ability to plan - your unit plan, impact - evidence of student learning) onto Canvas? If so, which ones have you done, which ones have you not done?

Part C. Closure

1. How are you practicing self-care?

2. What are your current struggles?

3. What concerns do you have for yourself/your loved ones?

4. What do you need from me (your Academic Clinical Educator)?

5. How have you focused on your students' learning?

\section{References}

Griggs, B., Sullivan-Losey, D., \& Zollman, A. (2018). More swim-less sink: Co-teaching advantages for middle and secondary teacher candidates. In J. Hollenbeck (Ed.), The American process: Uniting all in one (2nd ed., pp. 153-157)). Dubuque, IA: Kendall Hunt.

Maslow, A. (1954). Motivation and personality. New York, NY: Harper. 